\title{
Diverse Keyphrase Generation with Neural Unlikelihood Training
}

\author{
Hareesh Bahuleyan $^{\dagger}$ Layla El Asri ${ }^{\dagger}$ \\ ${ }^{\dagger}$ Borealis AI, Montreal, Canada \\ layla.elasri@borealisai.com \\ hareeshbahuleyan@gmail.com
}

\begin{abstract}
In this paper, we study sequence-to-sequence (S2S) keyphrase generation models from the perspective of diversity. Recent advances in neural natural language generation have made possible remarkable progress on the task of keyphrase generation, demonstrated through improvements on quality metrics such as $F_{1}$-score. However, the importance of diversity in keyphrase generation has been largely ignored. We first analyze the extent of information redundancy present in the outputs generated by a baseline model trained using maximum likelihood estimation (MLE). Our findings show that repetition of keyphrases is a major issue with MLE training. To alleviate this issue, we adopt neural unlikelihood (UL) objective for training the S2S model. Our version of UL training operates at (1) the target token level to discourage the generation of repeating tokens; (2) the copy token level to avoid copying repetitive tokens from the source text. Further, to encourage better model planning during the decoding process, we incorporate $K$-step ahead token prediction objective that computes both MLE and UL losses on future tokens as well. Through extensive experiments on datasets from three different domains we demonstrate that the proposed approach attains considerably large diversity gains, while maintaining competitive output quality. ${ }^{1}$
\end{abstract}

\section{Introduction}

Automatic keyphrase generation is the task of generating single or multi-word lexical units that provides readers with high level information about the key ideas or important topics described in a given source text. Apart from an information summarization perspective, this task has applications in various downstream natural language processing tasks such as text classification (Liu et al., 2009), document clustering (Hammouda et al., 2005) and information retrieval (Nguyen and Kan, 2007a).

Traditionally, keyphrases (KPs) were extracted from source documents by retrieving and ranking a set of candidate phrases through rule based approaches. With recent advances in neural natural language generation and availability of larger training corpora, this problem is formulated under a sequence-tosequence (S2S) modelling framework (Sutskever et al., 2014). This approach has an advantage that it can generate new and meaningful keyphrases which may be absent in the source text. The earliest work in this direction was by Meng et al. (2017), who train a S2S model to generate one keyphrase at a time. At inference time, they decode with beam sizes as high as 200, to generate a large number of KPs and finally de-duplicate the outputs. However, this is computationally expensive and wasteful because only $<5 \%$ of such KPs were found to be unique (Yuan et al., 2020).

An alternative approach is to train a S2S model to generate multiple keyphrases in a sequential manner, where the output KPs are separated by a pre-defined delimiter token. This method has an added benefit that the model automatically learns to generate a variable number of keyphrases depending on the input, instead of a user-specified fixed number of keyphrases (top- $k$ ) from a large list of candidate outputs. However, some previous approaches (Yuan et al., 2020) still use exhaustive beam search decoding to over-generate KPs and then apply post-processing to remove repetitions. Apart from the additional computational requirements, we argue that this method of avoiding information redundancy is a last-minute solution.

\footnotetext{
${ }^{1}$ Code is available at https://github.com/BorealisAI/keyphrase-generation

This work is licensed under a Creative Commons Attribution 4.0 International License. License details: http:// creativecommons.org/licenses/by/4.0/
} 


\begin{tabular}{|l|l|}
\hline Title & semi automated schema integration with sasmint \\
\hline Abstract & $\begin{array}{l}\text { the emergence of increasing number of collaborating organizations has made clear the need for supporting } \\
\text { interoperability infrastructures, enabling sharing and exchange of data among organizations . schema } \\
\text { matching and schema integration are the crucial components of the interoperability infrastructures } \\
\text { and their semi automation to interrelate or integrate heterogeneous and autonomous databases in } \\
\text { collaborative networks is desired . the semi automatic schema matching and integration sasmint system } \\
\text { introduced in this paper identifies and resolves }(. . .)\end{array}$ \\
\hline Ground Truth & schema integration; collaboration; schema matching; heterogeneity; data sharing \\
\hline MLE Baseline & $\begin{array}{l}\text { schema integration; sasmint ; schema matching ; schema integration ; schema matching ; sasmint } \\
\text { derivation markup language }\end{array}$ \\
\hline DivKGen & schema integration; interoperability infrastructures ; schema matching; sasmint \\
\hline
\end{tabular}

Table 1: Comparison of sample outputs generated by our model (DivKGen) vs. an MLE baseline. The repeating keyphrases are shown in red.

In this paper, we take a principled direction towards addressing the information redundancy issue in keyphrase generation models. We propose to tackle this problem directly during the training stage, rather than applying adhoc post-processing at inference time. Specifically, we adopt the neural unlikelihood training (UL) objective (Welleck et al., 2020), whereby the decoder is penalized for generating undesirable tokens. Welleck et al. (2020) introduce unlikelihood training for a language model setting. Since we work with a S2S setup, our version of UL loss consists of two components: (1) a target token level UL loss based on the target vocabulary to penalize the model for generating repeating tokens; (2) a copy token level UL loss based on the dynamic vocabulary of source tokens required for copy mechanism (Gu et al., 2016; See et al., 2017), which penalizes the model for copying repetitive tokens.

S2S models trained with maximum likelihood estimation (MLE) are usually tasked with the next token prediction objective. However, this does not necessarily incentivize the model to plan for future token prediction ahead of time. We observe such lack of model planning capability in our initial experiments with MLE models and to overcome this issue we propose to use $K$-step ahead token prediction. This modified training objective encourages the model to learn to correctly predict not just the current token, but also tokens upto $K$-steps ahead in the future. We then naturally incorporate UL training on the $K$-step ahead token prediction task.

We summarize our contributions as follows: (1) To improve the diversity of generated keyphrases in a principled manner during training, we adopt the unlikelihood objective for the S2S setting and propose a novel copy token unlikelihood loss. (2) In order to incentivize model planning, we augment our training objective function to incorporate $K$-step ahead token prediction. Additionally, we also introduce the $K$-step ahead unlikelihood losses. (3) We propose new metrics for benchmarking keyphrase generation models on diversity criterion. We carry out experiments on datasets from three different domains (scientific articles, news and community QA) and validate the effectiveness of our approach. We observe substantial gains in diversity while maintaining competitive output quality.

\section{Background and Motivation}

\subsection{Problem Definition}

The task of keyphrase generation can be formulated in the following manner. Given a source document $\mathbf{x}$, we are required to generate a set of keyphrases $\mathcal{Y}=\left\{\mathbf{y}^{1}, \mathbf{y}^{2}, \ldots, \mathbf{y}^{|\mathcal{Y}|}\right\}$ that best describe the input. The source document is denoted as a sequence of $S$ words: $\mathbf{x}=\left(x_{1}, x_{2}, \ldots, x_{S}\right)$. Each target keyphrase $\mathbf{y}^{i}=\left(y_{1}, y_{2}, \ldots, y_{T_{i}}\right)$ is also a word sequence of length $T_{i}$.

We follow the modelling setup adopted in previous work on keyphrase generation (Ye and Wang, 2018; Chan et al., 2019). Given document-keyphrases pair (x, Y), we concatenate all the ground truth keyphrases into a single linearized output sequence $\mathbf{y}=\mathbf{y}^{1} \diamond \mathbf{y}^{2} \diamond \ldots \diamond \mathbf{y}^{|\mathcal{Y}|}$, where $\diamond$ denotes a special delimiter token that is inserted in between consecutive keyphrases. The training data now consists of $(\mathrm{x}, \mathrm{y})$ pairs and one can conveniently use a sequence-to-sequence (S2S) modelling architecture to learn the mapping from $\mathbf{x}$ to $\mathbf{y}$. 


\subsection{Sequence Encoder-Decoder}

A bi-directional LSTM encoder (Hochreiter and Schmidhuber, 1997) reads the variable length source sequence $\mathbf{x}=\left(x_{1}, \ldots, x_{i}, \ldots, x_{S}\right)$ and produces a sequence of hidden state representations $h=$ $\left(h_{1}, \ldots, h_{i}, \ldots, h_{S}\right)$ with $h_{i} \in \mathbb{R}^{d_{h}}$, using the operation $h_{i}=f_{\text {enc }}\left(x_{i}, h_{i-1}\right)$ where $f_{\text {enc }}$ is a differentiable non-linear function.

For the decoder, we use a uni-directional LSTM which computes a hidden state $s_{t} \in \mathbb{R}^{d_{s}}$ at each decoding time step based on a non-linear function defined as $s_{t}=f_{d e c}\left(e_{t-1}, s_{t-1}\right)$. At training time, $e_{t-1}$ is the embedding of the ground truth previous word and at inference time, it is the embedding of the word predicted at the previous time step.

\subsection{Attention Guided Decoding}

By incorporating global attention mechanism (Bahdanau et al., 2015) into the basic S2S architecture, it is possible to dynamically align source information with the target hidden states during the decoding process. This is achieved by computing an alignment score between the decoder hidden state $s_{t}$ and each of the encoder hidden representations $\left\{\boldsymbol{h}_{i}\right\}_{i=1}^{S}$. At decoding time step $t$, this corresponds to

$$
\alpha_{t i}=\frac{\exp \left\{\tilde{\alpha}_{t i}\right\}}{\sum_{i^{\prime}=1}^{S} \exp \left\{\tilde{\alpha}_{t i^{\prime}}\right\}} ; \text { where } \tilde{\alpha}_{t i}=s_{t} \mathbf{W}_{a} h_{i}
$$

where $\alpha_{t i}$ is referred to as the attention probability score and $\mathbf{W}_{a}$ is a learnable attention weight matrix. Next we compute the attention context vector as a weighted summation across source hidden states.

$$
c_{t}=\sum_{i=1}^{S} \alpha_{t i} h_{i}
$$

Finally, the probability distribution over a predefined vocabulary $\mathcal{V}_{\text {Target }}$ of target tokens is obtained as

$$
P_{\text {target }}\left(y_{t}\right)=\operatorname{softmax}\left(\mathbf{W}_{v} \tilde{s}_{t}\right) ; \text { where } \tilde{s}_{t}=\tanh \left(\mathbf{W}_{u}\left[s_{t} \oplus c_{t}\right]\right)
$$

where $\oplus$ refers to the concatenation operator. Note that $\mathbf{W}_{u}$ and $\mathbf{W}_{v}$ are trainable decoder parameters and $y_{t} \in \mathcal{V}_{\text {Target }}$. For notational brevity, we omit the bias terms.

\subsection{Copy Mechanism}

We incorporate copy mechanism (Gu et al., 2016) to alleviate the out-of-vocabulary issue during generation, by allowing the decoder to selectively copy tokens from the source document. Specifically, we employ a learnable switching parameter $p_{\text {gen }}=\operatorname{sigmoid}\left(\mathbf{W}_{c}\left[s_{t} ; c_{t} ; e_{t-1}\right]\right)$ which refers to the probability of generating a token from the target vocabulary $\mathcal{V}_{\text {Target }}$. Thus, $\left(1-p_{\text {gen }}\right)$ corresponds to the probability of copying a token present on the source side whose dynamic vocabulary is denoted by $\mathcal{V}_{\mathbf{x}}$. The generation probability and the copy probability at time step $t$ are then combined to predict the next token as follows:

$$
P\left(y_{t}\right)=p_{\text {gen }} P_{\text {target }}\left(y_{t}\right)+\left(1-p_{\text {gen }}\right) P_{\text {copy }}\left(y_{t}\right)
$$

where $y_{t} \in \mathcal{V}_{\text {Target }} \cup \mathcal{V}_{\mathbf{x}}$ and $P_{\text {copy }}\left(y_{t}\right)=\sum_{i: x_{i}=y_{t}} \alpha_{t i}$ is the copy probability of token $y_{t}$ defined as a sum of its attention weights across all its occurrences in the source text.

\subsection{Maximum Likelihood Training}

Encoder-decoder models for sequence generation are typically trained using Maximum Likelihood Estimation (MLE). Concretely, for a given instance in the training data, MLE objective corresponds to learning the model parameters $\boldsymbol{\theta}$ that minimizes the negative log-likelihood loss defined as follows:

$$
\mathcal{L}_{\mathrm{MLE}}=-\sum_{t=1}^{L} \log P\left(y_{t} \mid \mathbf{y}_{1: t-1}, \mathbf{x}, \boldsymbol{\theta}\right)
$$

where $y_{t}$ is the $t$-th token in the ground truth output sequence $\mathbf{y}$ whose total length is $L$ tokens.

We begin with a setup where the S2S model for keyphrase generation is trained using MLE. We carry out preliminary experiments analyzing the diversity of the generation process and demonstrate the shortcomings of MLE-based training (Section 2.6) which paves way for the proposed approach (Section 3). 


\begin{tabular}{l|c|c|c}
\hline & \#Keyphrases & \% duplicate keyphrases & \% duplicate tokens \\
\hline Ground Truth & 5.3 & 0.1 & 7.3 \\
MLE Baseline & 7.3 & 26.6 & 36.0 \\
\hline
\end{tabular}

Table 2: A pilot study on KP $20 \mathrm{k}$ dataset validates our hypothesis about MLE-based training, which tends to generate a large number of repetitions in its outputs. The reported numbers are obtained by averaging the metrics across the test set.

\subsection{Lack of Diversity Issue}

We conduct a pilot study using KP $20 \mathrm{k}$ dataset (Meng et al., 2017), a corpus of scientific articles. Each article consists of a title, an abstract and a set of associated keyphrases. Table 1 shows one such example, along with outputs from two systems - a S2S model trained purely with MLE objective and our proposed model which is trained with a combination of unlikelihood training and future token prediction. It can be observed that with MLE objective alone, the S2S model tends to generate the same keyphrase over and over again. On the other hand, the output keyphrases from the proposed model summarizes the abstract of the scientific article, without any repetitions.

Furthermore, in Table 2 we quantify this lack of diversity issue using two simple metrics - the percentage of duplicate keyphrases and the percentage of duplicate tokens. On average, for an MLE model, about 27\% of the generated KPs and 36\% of the generated tokens are duplicates. These values are much higher than the percentage of repetitions present in the ground truth data. This implies that a significant computational effort is spent in the generation of redundant information. Moreover, additional post-processing pipelines are required in order to get rid of these repetitions. From a user experience point of view, the developed system should generate high quality KPs that describe the main ideas in the source text, without any information redundancy. We design our system keeping this objective in mind.

\section{Proposed Approach}

Rather than addressing the information redundancy issue through post-processing, we take a principled approach in this direction during training itself. Firstly, we adopt neural unlikelihood training (Welleck et al., 2020) for sequence-to-sequence setting by directly penalizing the decoder for either generating or copying repeating tokens. Secondly, we improve the planning capability of the decoder by incorporating a $K$-step ahead token prediction loss. This is achieved by using the same decoder hidden state but different attention mechanisms to decide which source tokens to be attended to, for predicting the target at the current time step, 1-step ahead and so on. An illustration of our approach is presented in Figure 1.

\subsection{Target Token Unlikelihood Loss}

The goal of unlikelihood training is to suppress the model's tendency to assign high probabilities to unnecessary tokens. During decoding, say at time step $t$, we maintain a negative candidate list $\mathcal{C}_{\text {Target }}^{t}$ that consists of tokens that should ideally be assigned a low probability for the current time step prediction. Formally, given $\mathcal{C}_{\text {TargetUL }}^{t}=\left\{c_{1}, \ldots, c_{m}\right\}$ where $c_{j} \in \mathcal{V}_{\text {Target }}$, we define the unlikelihood loss based on the target vocabulary across all time steps as follows

$$
\mathcal{L}_{\text {TargetUL }}=-\sum_{t=1}^{L} \sum_{c \in \mathcal{C}_{\text {Target }}^{t}} \log \left(1-P_{\text {target }}\left(c \mid \mathbf{y}_{1: t-1}, \mathbf{x}, \boldsymbol{\theta}\right)\right)
$$

Intuitively, assigning a high probability to a negative candidate token leads to a larger loss. Following Welleck et al. (2020), our negative candidate list for $\mathcal{L}_{\text {TargetUL }}$ consists of the ground truth context tokens from the previous time steps, i.e., $\mathcal{C}_{\text {Target }}^{t}=\left\{y_{1}, \ldots, y_{t-1}\right\} \backslash\left\{y_{t}\right\}$. In this manner, we effectively discourage the model from repeatedly generating tokens that are already present in the previous contexts.

\subsection{Copy Token Unlikelihood Loss}

In contrast to Welleck et al. (2020) who introduce UL training for language model setting, our application employs this method for a S2S task. As described in Section 2.4, our decoder utilizes a copy mechanism 

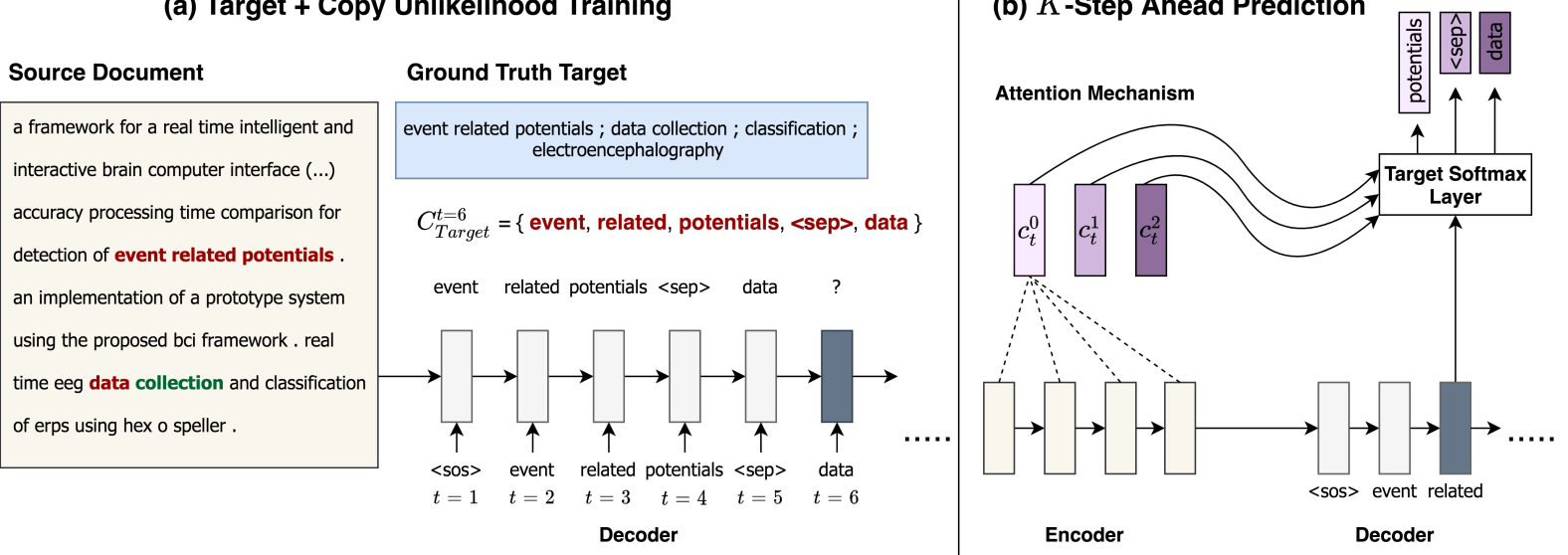

Figure 1: (a) Illustration of unlikelihood training. In the above example, at decoding time step $t=6$, the previous tokens from the target context form the negative candidate list denoted by $\mathcal{C}_{\text {Target }}^{t=6}$. The Target UL loss is computed using the probabilities assigned to these tokens. Similarly, the Copy UL loss discourages the model from copying the words displayed in red from the source document at $t=6$. Ideally, we would like the model to copy the word 'collection'. (b) Depiction of $K$-step ahead prediction with $K=2$. Different attention matrices are used to compute the corresponding attention context vectors for $k=0,1,2$. These are then individually fed to the final softmax layer along with the shared decoder hidden state to predict the token at the respective $k$. Copy mechanism is omitted from Figure 1(b) for simplicity.

that dynamically creates an extended vocabulary during generation based on the source tokens $\left(\mathcal{V}_{x}\right)$. An undesirable side-effect of copying is that the model might repeatedly attend to (and copy) the same set of source tokens over multiple decoding time steps, leading to repetitions in the output. To circumvent this issue, we propose an approach that we refer to as copy token unlikelihood loss.

For penalizing unnecessary copying, our negative candidate list at each time step is composed of ground truth context tokens from previous time steps that also appear in the source text (and thus can be copied).

$$
\mathcal{L}_{\text {CopyUL }}=-\sum_{t=1}^{L} \sum_{c \in \mathcal{C}_{\text {Copy }}^{t}} \log \left(1-P_{\text {copy }}\left(c \mid \mathbf{y}_{1: t-1}, \mathbf{x}, \boldsymbol{\theta}\right)\right)
$$

where $\mathcal{C}_{\text {Copy }}^{t}=\left\{y_{i} \mid y_{i} \in\left\{y_{1}, \ldots, y_{t-1}\right\} \backslash\left\{y_{t}\right\}\right.$ and $\left.y_{i} \in \mathcal{V}_{\mathbf{x}}\right\}$ and $P_{\text {copy }}(c \mid$.$) refers to the probability$ of copying a given token $c$ determined by the attention mechanism over the source tokens (Section 2.4).

\section{3 $\quad K$-Step Ahead Token Prediction Loss}

Keyphrases are made up of one or more tokens. The decoder in S2S models is usually tasked with simply predicting the next token given the context so far. This greedy approach does not incentivize the model to plan for the upcoming future tokens ahead of time. We mitigate this issue by directly incorporating the prediction of tokens $K$-steps ahead from the current time step into our training objective. To do so, we start with Equation 5, the MLE-based objective for next token prediction at time step $t$. This can be generalized for the prediction of upto $K$ tokens ahead in time as follows:

$$
\mathcal{L}_{K-\text { StepMLE }}=-\sum_{t=1}^{L} \sum_{k=0}^{K} \gamma_{k} \log P\left(y_{t+k} \mid \mathbf{y}_{1: t-1}, \mathbf{x}, \boldsymbol{\theta}\right)
$$

where $\gamma_{k}$ refers to the coefficient of the $k$ th step ahead token prediction loss. Note that the next token prediction MLE objective in Equation 5 is a special case of Equation 8 where $K=0$ and $\gamma_{0}=1.0$. One can think of the $K$-step ahead losses as a way to reward the model to plan the surface realization of the output sequence ahead of time. Intuitively, it makes sense to assign a high weightage to current token 
prediction (i.e., for $k=0$ ) and relatively downweight the losses incurred from future token predictions. We accomplish this by decaying the coefficient $\gamma_{k}$ using the formula $\gamma_{k}=\frac{1.0}{k+1}$.

For $K$-Step ahead prediction, we consider two implementation choices: (1) For each $k$, learn a different transformation $\mathbf{W}_{v}^{k}$ (in Equation 3) from the hidden representation to the logits over the vocabulary $\mathcal{V}_{\text {Target }}$. However, this increases the number of model parameters by $k \times d_{s_{t}} \times\left|\mathcal{V}_{\text {Target }}\right|$ where $d_{s_{t}}$ is the decoder hidden size. (2) With the second option, for each $k$, a different attention weight matrix $\mathbf{W}_{a}^{k}$ is learnt, while having a shared output transformation layer based on $\mathbf{W}_{v}$. More specifically, Equations 1 and 2 can be re-written as

$$
\tilde{\alpha}_{t i}^{k}=s_{t} \mathbf{W}_{a}^{k} h_{i} \quad ; \quad \alpha_{t i}^{k}=\frac{\exp \left\{\tilde{\alpha}_{t i}^{k}\right\}}{\sum_{i^{\prime}=1}^{S} \exp \left\{\tilde{\alpha}_{t i^{\prime}}^{k}\right\}} \quad ; \quad c_{t}^{k}=\sum_{i=1}^{S} \alpha_{t i}^{k} h_{i}
$$

The intuition behind such a formulation is that the different attention mechanisms (for different $k$ 's) learn different weighting schemes over the source tokens that enable the prediction of the future token at time step $t+k$. Moreover, this is much more parameter efficient because the number of extra parameters introduced into the model is only $k \times d_{s_{t}} \times d_{s_{t}}$, where $d_{s_{t}} \ll\left|\mathcal{V}_{\text {Target }}\right|$. Hence, we adopt the second implementation choice in our experiments.

\section{4 $\quad K$-Step Ahead Unlikelihood Loss}

In Section 3.3, we introduce an MLE-based loss for the task of $K$-step ahead token prediction. This idea can be naturally extended to the unlikelihood setting. Concretely, we impose the target and copy unlikelihood losses on the $K$-step ahead token prediction task as follows:

$$
\begin{aligned}
\mathcal{L}_{K-\text { StepTargetUL }} & =-\sum_{t=1}^{L} \sum_{k=0}^{K} \gamma_{k} \sum_{c \in \mathcal{C}_{\text {Target }}^{t+k}} \log \left(1-P_{\text {target }}\left(c \mid \mathbf{y}_{1: t-1}, \mathbf{x}, \boldsymbol{\theta}\right)\right) \\
\mathcal{L}_{K \text {-StepCopyUL }} & =-\sum_{t=1}^{L} \sum_{k=0}^{K} \gamma_{k} \sum_{c \in \mathcal{C}_{\text {Copy }}^{t+k}} \log \left(1-P_{\text {copy }}\left(c \mid \mathbf{y}_{1: t-1}, \mathbf{x}, \boldsymbol{\theta}\right)\right)
\end{aligned}
$$

where the negative candidate lists are $\mathcal{C}_{\text {Target }}^{t+k}=\left\{y_{1}, \ldots, y_{t+k-1}\right\} \backslash\left\{y_{t+k}\right\}$ and $\mathcal{C}_{\text {Copy }}^{t+k}=\left\{y_{i} \mid y_{i} \in\right.$ $\left\{y_{1}, \ldots, y_{t+k-1}\right\} \backslash\left\{y_{t+k}\right\}$ and $\left.y_{i} \in \mathcal{V}_{\mathbf{x}}\right\}$. Penalizing the model for future repetitions through the $K$-step ahead unlikelihood losses should further enhance overall diversity of its outputs.

\subsection{Overall Training Objective}

To summarize, our S2S model is trained with a combination of likelihood and unlikelihood losses on the current $(k=0)$ and future $(k=1, \ldots, K)$ token prediction tasks. The overall loss function is given by:

$$
\mathcal{L}=\mathcal{L}_{K \text {-StepMLE }}+\lambda_{T} \mathcal{L}_{K \text {-StepTargetUL }}+\lambda_{C} \mathcal{L}_{K-\text { StepCopyUL }}
$$

where $\lambda_{T}$ and $\lambda_{C}$ are hyperparameters that control the weight of target and copy UL losses respectively.

\section{Experiment Setup}

Evaluation Metrics. To measure the quality of generated keyphrases, i.e., its relevance with respect to the source document, we compare the generated set of KPs to the KPs in the corresponding ground truth data. To this end, we report $F_{1} @ M$, where $M$ refers to the number of model predicted keyphrases. We also include the corresponding precision and recall metrics. As justified in previous work (Chan et al., 2019; Yuan et al., 2020), $F_{1} @ M$ captures the ability of abstractive S2S models in generating a variable number of KPs depending on the source document, in comparison to traditional extractive methods where one is required to specify a cutoff in order to output the top- $k$ keyphrases. However, different from previous work, we report the overall $F_{1} @ M$ score rather than separately computing this score for keyphrases present vs. absent in the source text. This is because our goal in this work is to 
overcome the lack of diversity issue in keyphrase generation models, and not necessarily to generate more absent keyphrases.

In order to evaluate the model outputs on the criterion of diversity, we define the following metrics:

\% Duplicate KPs $=\left(1-\frac{\text { Number of Unique Keyphrases }}{\text { Total Number of Generated Keyphrases }}\right) * 100$
$\%$ Duplicate Tokens $=\left(1-\frac{\text { Number of Unique Tokens }}{\text { Total Number of Generated Tokens }}\right) * 100$

\# KPs: We report the number of keyphrases generated. Ideally, the model should generate the same number of keyphrases as present in the ground truth target sequence.

The next three metrics measure the inter-keyphrase similarity among the generated set of keyphrases - a lower value indicates fewer repetitions and thus more diversity in the output.

Self-BLEU: We use Self-BLEU (Zhu et al., 2018) which computes pairwise BLEU score (Papineni et al., 2002) between generated KPs. This metric captures word level surface overlap.

EditDist $^{2}$ : String matching can also be carried out at the character level. Through our EditDist metric, we calculate the pairwise Levenshtein Distance between KPs output by the model.

EmbSim: With Self-BLEU and EditDist, we can only capture surface level repetitions between KPs. To overcome this limitation, we propose to use pre-trained phrase-level embeddings that measures inter-keyphrase similarity at a semantic level. Specifically, we compute pairwise cosine similarities between Sent2Vec embedding representations (Pagliardini et al., 2018) of keyphrases. Sent2Vec has been reported to perform well in previous work on keyphrase extraction (Bennani-Smires et al., 2018).

All reported metrics are computed for each test set output, followed by averaging across all records.

Datasets. We carry out experiments on datasets from three domains ${ }^{3}$ : (1) KP $20 \mathrm{~K}$ (Meng et al., 2017) is a dataset of scientific articles; (2) KPTimes (Gallina et al., 2019) consists of news articles and editor assigned keyphrases; (3) StackEx (Yuan et al., 2020) is a dataset curated from a community question answering forum with keyphrases being the user assigned tags.

Baselines. We compare our approach to five S2S keyphrase generation baselines ${ }^{4}$ (4 MLE-based models and 1 which uses a reinforcement learning objective) - (1) catSeq: A S2S model trained solely using the MLE objective. (refer to Equation 5). (2) catSeqD: Introduced by Yuan et al. (2020), this method uses auxiliary semantic coverage and orthogonality losses to enhance generation diversity. (3) catSeqCorr: Chen et al. (2018) augment the attention scheme in the catSeq model with a coverage module and review mechanism. (4) catSeqTG: Instead of simply concatenating the article title and abstract together to form the source document, Chen et al. (2019b) design a model architecture that separately encodes the title information using an attention-guided matching layer. (5) catSeqTG-2RF1: Chan et al. (2019) extend the catSeqTG model by training it using a reinforcement learning (RL) objective where $F_{1}$-score is directly used as the reward. Implementation details of our model are provided in Appendix B.

\section{Results and Analysis}

We report quality and diversity metrics on five baselines and three variants of the proposed approach (Table 3). We refer to our model as DivKGen; the base UL variant is trained with the regular MLE objective plus target and copy level unlikelihood losses. The rows denoted by $+K$-StepMLE and $+K$ StepUL are variants built on top of the base variant, by cumulatively incorporating $K$-Step ahead token prediction MLE and $K$-Step ahead UL losses respectively. For each dataset, we also report the ground truth statistics. For instance, the KP2OK has an average keyphrase count of 5.3 in the test set with only $0.1 \%$ duplicate KPs and 7.3\% duplicate tokens. In comparison, the MLE baseline (catSeq) produces a much

\footnotetext{
${ }^{2}$ We utilize the fuzzywuzzy library in Python: https://github.com/seatgeek/fuzzywuzzy. It computes a score between 0 and 100, where 100 means exactly matching keyphrases.

${ }^{3}$ Additional results on five datasets that only provide a test set for evaluation (INSPEC, KRAP IVIN, NUS, SEMEVAL, DUC) are provided in the Appendix C.

${ }^{4}$ We use the open-source code provided by Chan et al. (2019) for implementing the baselines: https://github.com/ kenchan0226/keyphrase-generation-rl
} 


\begin{tabular}{|c|c|c|c|c|c|c|c|c|c|c|}
\hline & & \multicolumn{3}{|c|}{ Quality Evaluation } & \multicolumn{6}{|c|}{ Diversity Evaluation } \\
\hline & & $P @ M$ & $R @ M$ & $F_{1} @ M$ & \#KPs & $\begin{array}{c}\text { \%Duplicate } \\
\text { KPs } \downarrow\end{array}$ & $\begin{array}{c}\text { \%Duplicate } \\
\text { Tokens } \downarrow\end{array}$ & $\begin{array}{c}\text { Self- } \\
\text { BLEU } \downarrow\end{array}$ & $\begin{array}{c}\text { Edit- } \\
\text { Dist } \downarrow\end{array}$ & $\begin{array}{l}\text { Emb- } \\
\text { Sim } \downarrow\end{array}$ \\
\hline \multirow{9}{*}{ 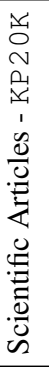 } & Ground Truth & - & - & - & $\rightarrow 5.3$ & 0.1 & 7.3 & 3.8 & 32.7 & 0.159 \\
\hline & catSeq & 0.291 & 0.260 & 0.274 & 7.3 & 26.6 & 36.0 & 26.6 & 45.6 & 0.328 \\
\hline & catSeqD & 0.294 & 0.257 & 0.274 & 6.7 & 25.7 & 35.3 & 27.0 & 45.3 & 0.325 \\
\hline & catSeqCorr & 0.283 & 0.264 & 0.273 & 7.0 & 23.2 & 33.5 & 24.5 & 44.0 & 0.309 \\
\hline & catSeqTG & 0.295 & 0.262 & 0.278 & 6.8 & 24.7 & 34.3 & 26.2 & 45.2 & 0.323 \\
\hline & catSeqTG-2RF1 & 0.274 & 0.286 & 0.280 & 7.5 & 30.9 & 41.7 & 30.7 & 46.7 & 0.341 \\
\hline & DivKGen (UL) & 0.277 & 0.261 & 0.269 & 5.0 & 5.3 & 12.6 & 9.7 & 34.4 & 0.181 \\
\hline & $+K$-StepMLE & 0.274 & 0.239 & 0.255 & 4.6 & 6.1 & 13.9 & 11.5 & 36.2 & 0.197 \\
\hline & $+K$-StepUL & 0.273 & 0.240 & 0.256 & 4.6 & 4.9 & 11.7 & 8.8 & 35.2 & 0.185 \\
\hline \multirow{9}{*}{ 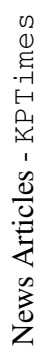 } & Ground Truth & - & - & - & $\rightarrow 5.0$ & 0.1 & 4.9 & 2.2 & 26.5 & 0.135 \\
\hline & catSeq & 0.399 & 0.375 & 0.387 & 5.9 & 13.7 & 20.7 & 17.2 & 32.7 & 0.202 \\
\hline & catSeqD & 0.395 & 0.374 & 0.384 & 6.2 & 15.8 & 22.6 & 18.3 & 33.5 & 0.212 \\
\hline & catSeqCorr & 0.397 & 0.376 & 0.386 & 5.6 & 10.3 & 17.6 & 13.8 & 31.6 & 0.190 \\
\hline & catSeqTG & 0.402 & 0.380 & 0.391 & 5.9 & 13.8 & 21.2 & 17.6 & 32.8 & 0.203 \\
\hline & catSeqTG-2RF1 & 0.389 & 0.386 & 0.387 & 6.0 & 14.0 & 21.0 & 18.6 & 32.5 & 0.192 \\
\hline & DivKGen (UL) & 0.385 & 0.320 & 0.350 & 4.3 & 2.3 & 7.0 & 4.2 & 27.8 & 0.142 \\
\hline & $+K$-StepMLE & 0.391 & 0.316 & 0.349 & 4.3 & 3.3 & 7.9 & 5.3 & 27.9 & 0.147 \\
\hline & $+K$-StepUL & 0.371 & 0.314 & 0.340 & 4.6 & 3.6 & 8.7 & 5.8 & 28.3 & 0.149 \\
\hline \multirow{9}{*}{ 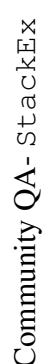 } & Ground Truth & - & - & - & $\rightarrow 2.7$ & 0.3 & 2.9 & 1.5 & 24.2 & 0.167 \\
\hline & catSeq & 0.526 & 0.518 & 0.522 & 2.7 & 4.3 & 7.4 & 4.1 & 28.2 & 0.226 \\
\hline & catSeqD & 0.510 & 0.524 & 0.517 & 2.8 & 5.0 & 8.6 & 4.8 & 28.8 & 0.230 \\
\hline & catSeqCorr & 0.501 & 0.526 & 0.513 & 2.9 & 5.4 & 9.3 & 5.2 & 29.1 & 0.235 \\
\hline & catSeqTG & 0.522 & 0.529 & 0.526 & 2.8 & 3.5 & 7.0 & 3.9 & 27.5 & 0.216 \\
\hline & catSeqTG-2RF1 & 0.433 & 0.570 & 0.492 & 3.8 & 6.7 & 11.8 & 6.2 & 29.0 & 0.220 \\
\hline & DivKGen (UL) & 0.512 & 0.453 & 0.481 & 2.2 & 0.3 & 1.4 & 0.5 & 23.3 & 0.175 \\
\hline & $+K$-StepMLE & 0.532 & 0.438 & 0.480 & 2.0 & 0.4 & 1.5 & 0.6 & 23.1 & 0.171 \\
\hline & $+K$-StepUL & 0.516 & 0.454 & 0.483 & 2.2 & 0.4 & 1.6 & 0.7 & 23.7 & 0.170 \\
\hline
\end{tabular}

Table 3: KP generation results on datasets from 3 domains, evaluated on both quality and diversity criteria.

higher percentage of repetitions. This is also evident from the inter-keyphrase pairwise similarity metrics Self-BLEU, EditDist and EmbSim. Surprisingly, the previous best performing model catSeqTG-2RF1, which uses RL to improve $F_{1}$ score, does worse than all MLE baselines in terms of diversity.

In contrast, DivKGen, our proposed approach achieves much better diversity than all baselines. The repetition percentages are lowered and are relatively closer to the ground truth. There is a large boost by simply adding token and copy UL losses to the baseline MLE model. For KP 20K dataset, we obtain small diversity gains through the incorporation of $K$-Step ahead losses whereas for the other two datasets, it does not result an improvement. A possible explanation is that the base DivKGen (UL) variant itself steers the diversity statistics to be quite close to that of the ground truth of these datasets. As a result, it becomes increasingly difficult to achieve a further reduction in this gap through any additional model changes.

With regards to quality evaluation metrics, it can be observed that DivKGen models have slightly lower scores. This can be explained from a quality-diversity trade-off viewpoint. As the model attempts to explore the output space through the generation of more interesting KPs, it may output new KPs that are not present in the ground truth, thus resulting in lower precision. DivKGen generates shorter sequences (and hence may not be able to produce all the KPs as per the ground truth) than the baselines, which could explain the lower recall.

Quality-Diversity Trade-off. We train different versions of DivKGen (UL) model on KP 20K dataset by varying $\lambda_{T}{ }^{5}$, the UL loss coefficient (refer Equation 12). As depicted in Figure 2, it can be seen that there is an obvious quality-diversity trade-off. For higher values of $\lambda_{T}$, we achieve a higher level of diversity

\footnotetext{
${ }^{5}$ For simplicity, we set $\lambda_{T}=\lambda_{C}$ to control the number of variable hyperparameters in the quality-diversity trade-off analysis.
} 


\begin{tabular}{l|c|c|c|c}
\hline DivKGen Variants & $\begin{array}{c}\text { Overall } \\
F_{1} @ M \uparrow\end{array}$ & $\begin{array}{c}\text { \%Duplicate } \\
\text { KPs } \downarrow\end{array}$ & $\begin{array}{c}\text { \%Duplicate } \\
\text { Tokens } \downarrow\end{array}$ & $\begin{array}{c}\text { Self- } \\
\text { BLEU } \downarrow\end{array}$ \\
\hline w/ TargetUL & $\mathbf{0 . 2 7 7}$ & 12.0 & 19.8 & 16.7 \\
w/ CopyUL & 0.263 & 14.1 & 22.7 & 19.9 \\
w/ K-StepMLE & 0.265 & 12.6 & 18.9 & 16.3 \\
w/ TargetUL + CopyUL & 0.269 & 5.3 & 12.6 & 9.7 \\
+ K-StepMLE & 0.255 & 6.1 & 13.9 & 11.5 \\
+ K-StepUL & 0.256 & $\mathbf{4 . 9}$ & $\mathbf{1 1 . 7}$ & $\mathbf{8 . 8}$ \\
\hline
\end{tabular}

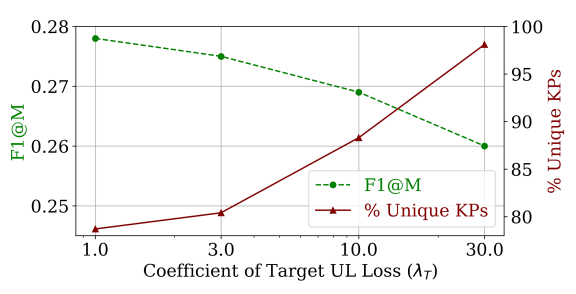

Table 4: Ablation study on the KP20k dataset. Each row denotes a DivKGen model variant obtained by adding the Figure 2: Illustration of qualitydiversity trade-off: \%Unique KPs = (100-\%Duplicate KPs) is used as a specified component. The last row corresponds to the full model.

representative metric for diversity.

(more unique KPs) at the cost of quality (and vice versa). Similar behaviour has been reported previously in the text generation literature (Bahuleyan et al., 2018; Gao et al., 2019). Hence, we recommend tuning the hyperparameters $\lambda_{T}$ and $\lambda_{C}$ to achieve a desired level of diversity.

Ablation Study. We conduct an ablation analysis to investigate the effect of different losses. We start with the MLE baseline and add loss components one-by-one as presented in Table 4. It is evident that the best diversity is obtained while using the full model (last row). Also, interestingly each individual loss component by itself (i.e., TargetUL, CopyUL and $K$-StepMLE), is not as effective as their combination. This suggests that each of the losses contribute in a synergetic manner to maximize diversity gains.

\section{Related Work}

Keyphrase Generation and Extraction. Traditional KP extraction methods such as TextRank (Mihalcea and Tarau, 2004) and TopicRank (Bougouin et al., 2013) first select candidate phrases from the source document using heuristics and then rank these candidates based on some measure of relevance or importance. Meng et al. (2017) formulate keyphrase generation as a S2S learning problem, with an advantage over previous extractive methods that it could even generate relevant KPs absent from the source text. A limitation of their approach is that one was still required to rank the top- $k$ KPs. This was addressed in works by (Ye and Wang, 2018) and (Yuan et al., 2020) which could generate a variable number of KPs depending on the input. We adopt a similar setup but carry out a comprehensive analysis of such models in terms of their output diversity, which has been largely ignored in previous work.

Diversity in Language Generation. Diversity promoting objectives for text generation have been previously explored in the literature (Li et al., 2016; Niu and Bansal, 2020; Jiang et al., 2020). However, these studies examine the overall corpus level diversity. For instance, the lack of diversity in a dialogue system, due to the fact that the model generates frequently seen responses from the training set. In our case, we address a different kind of diversity issue, arising as a result of repetitions occurring within individual outputs. Thus neural unlikelihood training (Welleck et al., 2020) is well suited to our problem. Test time decoding strategies to improve diversity such as top- $k$ sampling (Fan et al., 2018), nucleus sampling (Holtzman et al., 2020) and diverse beam search (Vijayakumar et al., 2018) are orthogonal to our approach and can naturally be incorporated.

\section{Conclusion and Future Work}

In this work, we first point out the shortcomings of MLE based training for keyphrase generation. We specifically address the lack of output diversity issue via the use of unlikelihood training objective. We adopt a target level unlikelihood loss and propose a novel copy token unlikelihood loss, the combination of which provides large diversity gains. In addition, a $K$-step ahead MLE and UL objective is incorporated into the training. Through extensive experiments on datasets from three different domains, we demonstrate the effectiveness of our model for diverse keyphrase generation. For future work, we plan to explore directions that would enable us to simultaneously optimize for quality and diversity metrics. 


\section{Acknowledgements}

We thank Francis Duplessis, Ivan Kobyzev, Jackie Chi Kit Cheung, Kushal Arora, Marjan Albooyeh, Mehran Kazemi, Simon Prince and Wenjie Zi for valuable inputs and helpful discussions.

\section{References}

Dzmitry Bahdanau, Kyunghyun Cho, and Yoshua Bengio. 2015. Neural machine translation by jointly learning to align and translate. In Yoshua Bengio and Yann LeCun, editors, 3rd International Conference on Learning Representations, ICLR 2015, San Diego, CA, USA, May 7-9, 2015, Conference Track Proceedings.

Hareesh Bahuleyan, Lili Mou, Olga Vechtomova, and Pascal Poupart. 2018. Variational attention for sequenceto-sequence models. In Proceedings of the 27th International Conference on Computational Linguistics, pages 1672-1682, Santa Fe, New Mexico, USA, August. Association for Computational Linguistics.

Kamil Bennani-Smires, Claudiu Musat, Andreea Hossmann, Michael Baeriswyl, and Martin Jaggi. 2018. Simple unsupervised keyphrase extraction using sentence embeddings. In Proceedings of the 22nd Conference on Computational Natural Language Learning, pages 221-229, Brussels, Belgium, October. Association for Computational Linguistics.

Adrien Bougouin, Florian Boudin, and Béatrice Daille. 2013. Topicrank: Graph-based topic ranking for keyphrase extraction. In International Joint Conference on Natural Language Processing (IJCNLP), pages 543-551.

Hou Pong Chan, Wang Chen, Lu Wang, and Irwin King. 2019. Neural keyphrase generation via reinforcement learning with adaptive rewards. In Proceedings of the 57th Annual Meeting of the Association for Computational Linguistics, pages 2163-2174, Florence, Italy, July. Association for Computational Linguistics.

Jun Chen, Xiaoming Zhang, Yu Wu, Zhao Yan, and Zhoujun Li. 2018. Keyphrase generation with correlation constraints. In Proceedings of the 2018 Conference on Empirical Methods in Natural Language Processing, pages 4057-4066.

Wang Chen, Hou Pong Chan, Piji Li, Lidong Bing, and Irwin King. 2019a. An integrated approach for keyphrase generation via exploring the power of retrieval and extraction. In Proceedings of the 2019 Conference of the North American Chapter of the Association for Computational Linguistics: Human Language Technologies, Volume 1 (Long and Short Papers), pages 2846-2856, Minneapolis, Minnesota, June. Association for Computational Linguistics.

Wang Chen, Yifan Gao, Jiani Zhang, Irwin King, and Michael R Lyu. 2019b. Title-guided encoding for keyphrase generation. In Proceedings of the AAAI Conference on Artificial Intelligence, volume 33, pages 6268-6275.

Angela Fan, Mike Lewis, and Yann Dauphin. 2018. Hierarchical neural story generation. In Proceedings of the 56th Annual Meeting of the Association for Computational Linguistics (Volume 1: Long Papers), pages 889-898, Melbourne, Australia, July. Association for Computational Linguistics.

Ygor Gallina, Florian Boudin, and Beatrice Daille. 2019. KPTimes: A large-scale dataset for keyphrase generation on news documents. In Proceedings of the 12th International Conference on Natural Language Generation, pages 130-135, Tokyo, Japan, October-November. Association for Computational Linguistics.

Xiang Gao, Sungjin Lee, Yizhe Zhang, Chris Brockett, Michel Galley, Jianfeng Gao, and Bill Dolan. 2019. Jointly optimizing diversity and relevance in neural response generation. In Proceedings of the 2019 Conference of the North American Chapter of the Association for Computational Linguistics: Human Language Technologies, Volume 1 (Long and Short Papers), pages 1229-1238, Minneapolis, Minnesota, June. Association for Computational Linguistics.

Matt Gardner, Joel Grus, Mark Neumann, Oyvind Tafjord, Pradeep Dasigi, Nelson F. Liu, Matthew Peters, Michael Schmitz, and Luke Zettlemoyer. 2018. AllenNLP: A deep semantic natural language processing platform. In Proceedings of Workshop for NLP Open Source Software (NLP-OSS), pages 1-6, Melbourne, Australia, July. Association for Computational Linguistics.

Jiatao Gu, Zhengdong Lu, Hang Li, and Victor O.K. Li. 2016. Incorporating copying mechanism in sequence-tosequence learning. In Proceedings of the 54th Annual Meeting of the Association for Computational Linguistics (Volume 1: Long Papers), pages 1631-1640, Berlin, Germany, August. Association for Computational Linguistics. 
Khaled M Hammouda, Diego N Matute, and Mohamed S Kamel. 2005. Corephrase: Keyphrase extraction for document clustering. In International workshop on machine learning and data mining in pattern recognition, pages 265-274. Springer.

Sepp Hochreiter and Jürgen Schmidhuber. 1997. Long short-term memory. Neural computation, 9(8):1735-1780.

Ari Holtzman, Jan Buys, Li Du, Maxwell Forbes, and Yejin Choi. 2020. The curious case of neural text degeneration. In 8th International Conference on Learning Representations, ICLR 2020, Addis Ababa, Ethiopia, April 26-30, 2020. OpenReview.net.

Anette Hulth and Beáta B. Megyesi. 2006. A study on automatically extracted keywords in text categorization. In Proceedings of the 21st International Conference on Computational Linguistics and 44th Annual Meeting of the Association for Computational Linguistics, pages 537-544, Sydney, Australia, July. Association for Computational Linguistics.

Shaojie Jiang, Thomas Wolf, Christof Monz, and Maarten de Rijke. 2020. TLDR: token loss dynamic reweighting for reducing repetitive utterance generation. CoRR, abs/2003.11963.

Su Nam Kim, Olena Medelyan, Min-Yen Kan, and Timothy Baldwin. 2010. Semeval-2010 task 5: Automatic keyphrase extraction from scientific articles. In Proceedings of the 5th International Workshop on Semantic Evaluation, pages 21-26.

Diederik P. Kingma and Jimmy Ba. 2015. Adam: A method for stochastic optimization. In Yoshua Bengio and Yann LeCun, editors, 3rd International Conference on Learning Representations, ICLR 2015, San Diego, CA, USA, May 7-9, 2015, Conference Track Proceedings.

Mikalai Krapivin, Aliaksandr Autaeu, and Maurizio Marchese. 2009. Large dataset for keyphrases extraction. Technical report, University of Trento.

Jiwei Li, Michel Galley, Chris Brockett, Jianfeng Gao, and Bill Dolan. 2016. A diversity-promoting objective function for neural conversation models. In Proceedings of the 2016 Conference of the North American Chapter of the Association for Computational Linguistics: Human Language Technologies, pages 110-119, San Diego, California, June. Association for Computational Linguistics.

Feifan Liu, Deana Pennell, Fei Liu, and Yang Liu. 2009. Unsupervised approaches for automatic keyword extraction using meeting transcripts. In Proceedings of Human Language Technologies: The 2009 Annual Conference of the North American Chapter of the Association for Computational Linguistics, pages 620-628, Boulder, Colorado, June. Association for Computational Linguistics.

Rui Meng, Sanqiang Zhao, Shuguang Han, Daqing He, Peter Brusilovsky, and Yu Chi. 2017. Deep keyphrase generation. In Proceedings of the 55th Annual Meeting of the Association for Computational Linguistics (Volume 1: Long Papers), pages 582-592, Vancouver, Canada, July. Association for Computational Linguistics.

Rui Meng, Xingdi Yuan, Tong Wang, Peter Brusilovsky, Adam Trischler, and Daqing He. 2019. Does order matter? an empirical study on generating multiple keyphrases as a sequence. CoRR, abs/1909.03590.

Rada Mihalcea and Paul Tarau. 2004. TextRank: Bringing order into text. In Proceedings of the 2004 Conference on Empirical Methods in Natural Language Processing, pages 404-411, Barcelona, Spain, July. Association for Computational Linguistics.

Thuy Dung Nguyen and Min-Yen Kan. 2007a. Keyphrase extraction in scientific publications. In International conference on Asian digital libraries, pages 317-326. Springer.

Thuy Dung Nguyen and Min-Yen Kan. 2007b. Keyphrase extraction in scientific publications. In International conference on Asian digital libraries, pages 317-326. Springer.

Tong Niu and Mohit Bansal. 2020. Avgout: A simple output-probability measure to eliminate dull responses. In The Thirty-Fourth AAAI Conference on Artificial Intelligence, AAAI 2020, The Thirty-Second Innovative Applications of Artificial Intelligence Conference, IAAI 2020, The Tenth AAAI Symposium on Educational Advances in Artificial Intelligence, EAAI 2020, New York, NY, USA, February 7-12, 2020, pages 8560-8567. AAAI Press.

Matteo Pagliardini, Prakhar Gupta, and Martin Jaggi. 2018. Unsupervised learning of sentence embeddings using compositional n-gram features. In Proceedings of the 2018 Conference of the North American Chapter of the Association for Computational Linguistics: Human Language Technologies, Volume 1 (Long Papers), pages 528-540, New Orleans, Louisiana, June. Association for Computational Linguistics. 
Kishore Papineni, Salim Roukos, Todd Ward, and Wei-Jing Zhu. 2002. Bleu: a method for automatic evaluation of machine translation. In Proceedings of the 40th annual meeting on association for computational linguistics, pages 311-318. Association for Computational Linguistics.

Adam Paszke, Sam Gross, Francisco Massa, Adam Lerer, James Bradbury, Gregory Chanan, Trevor Killeen, Zeming Lin, Natalia Gimelshein, Luca Antiga, Alban Desmaison, Andreas Köpf, Edward Yang, Zachary DeVito, Martin Raison, Alykhan Tejani, Sasank Chilamkurthy, Benoit Steiner, Lu Fang, Junjie Bai, and Soumith Chintala. 2019. Pytorch: An imperative style, high-performance deep learning library. In Hanna M. Wallach, Hugo Larochelle, Alina Beygelzimer, Florence d'Alché-Buc, Emily B. Fox, and Roman Garnett, editors, Advances in Neural Information Processing Systems 32: Annual Conference on Neural Information Processing Systems 2019, NeurIPS 2019, 8-14 December 2019, Vancouver, BC, Canada, pages 8024-8035.

Abigail See, Peter J. Liu, and Christopher D. Manning. 2017. Get to the point: Summarization with pointergenerator networks. In Proceedings of the 55th Annual Meeting of the Association for Computational Linguistics (Volume 1: Long Papers), pages 1073-1083, Vancouver, Canada, July. Association for Computational Linguistics.

Ilya Sutskever, Oriol Vinyals, and Quoc V Le. 2014. Sequence to sequence learning with neural networks. In Advances in neural information processing systems, pages 3104-3112.

Ashish Vaswani, Noam Shazeer, Niki Parmar, Jakob Uszkoreit, Llion Jones, Aidan N Gomez, Łukasz Kaiser, and Illia Polosukhin. 2017. Attention is all you need. In Advances in neural information processing systems, pages 5998-6008.

Ashwin K. Vijayakumar, Michael Cogswell, Ramprasaath R. Selvaraju, Qing Sun, Stefan Lee, David J. Crandall, and Dhruv Batra. 2018. Diverse beam search for improved description of complex scenes. In Sheila A. McIlraith and Kilian Q. Weinberger, editors, Proceedings of the Thirty-Second AAAI Conference on Artificial Intelligence, (AAAI-18), the 30th innovative Applications of Artificial Intelligence (IAAI-18), and the 8th AAAI Symposium on Educational Advances in Artificial Intelligence (EAAI-18), New Orleans, Louisiana, USA, February 2-7, 2018, pages 7371-7379. AAAI Press.

Xiaojun Wan and Jianguo Xiao. 2008. Single document keyphrase extraction using neighborhood knowledge. In $A A A I$, volume 8, pages 855-860.

Sean Welleck, Ilia Kulikov, Stephen Roller, Emily Dinan, Kyunghyun Cho, and Jason Weston. 2020. Neural text generation with unlikelihood training. In International Conference on Learning Representations.

Hai Ye and Lu Wang. 2018. Semi-supervised learning for neural keyphrase generation. In Proceedings of the 2018 Conference on Empirical Methods in Natural Language Processing, pages 4142-4153, Brussels, Belgium, October-November. Association for Computational Linguistics.

Xingdi Yuan, Tong Wang, Rui Meng, Khushboo Thaker, Peter Brusilovsky, Daqing He, and Adam Trischler. 2020. One size does not fit all: Generating and evaluating variable number of keyphrases. In Proceedings of the 58th Annual Meeting of the Association for Computational Linguistics, Seattle, USA. Association for Computational Linguistics.

Yaoming Zhu, Sidi Lu, Lei Zheng, Jiaxian Guo, Weinan Zhang, Jun Wang, and Yong Yu. 2018. Texygen: A benchmarking platform for text generation models. In The 41st International ACM SIGIR Conference on Research \& Development in Information Retrieval, pages 1097-1100. 


\section{Appendix}

\section{Diverse Keyphrase Generation with Neural Unlikelihood Training}

\section{A Dataset Statistics}

Table 5 presents the number of instances in each dataset, split across training, validatation and test sets.

\begin{tabular}{c|c|c|c}
\hline Dataset & \# Train & \#Validation & \#Test \\
\hline KP20K & $530 \mathrm{k}$ & $20 \mathrm{k}$ & $20 \mathrm{k}$ \\
KPTimes* & $260 \mathrm{k}$ & $10 \mathrm{k}$ & $20 \mathrm{k}$ \\
StackEx & $299 \mathrm{k}$ & $16 \mathrm{k}$ & $16 \mathrm{k}$ \\
\hline INSPEC & - & 1500 & 500 \\
SEMEVAL & - & 144 & 100 \\
KRAPIVIN & - & 1844 & 460 \\
NUS & - & - & 211 \\
DUC & - & - & 308 \\
\hline
\end{tabular}

Table 5: Train/validation/test statistics of the datasets used in this work.

*Note that our test set for KPTimes is a combination of 10k records from KPTimes and 10k records from JPTimes (Gallina et al., 2019).

\section{B Implementation Details}

We use the AllenNLP package (Gardner et al., 2018), which is built on PyTorch framework (Paszke et al., 2019), for implementing our models. We provide as input to the model the concatenated title and abstract. Following (Yuan et al., 2020), the ground truth target keyphrases are arranged as a sequence, where the absent KPs follow the present KPs. The size of source and target vocabularies are set to $50 \mathrm{k}$ and $10 \mathrm{k}$ respectively. The delimiter token that is inserted in between target keyphrases is denoted as $<\mathrm{SEP}\rangle$. Both the $\operatorname{LSTM}^{6}$ encoder and decoder have a hidden size of $100 \mathrm{~d}$. Word embeddings on both the source and target side are also set to 100d and randomly initialized. We use Adam optimizer (Kingma and Ba, 2015) with default parameters to train the model. The batch size is set to 64 and we incorporate early stopping based on validation $F_{1}$ score as the criterion.

Regarding the loss term coefficients for UL losses and $K$-step ahead loss, we set $\lambda_{T}=15.0, \lambda_{C}=18.0$ and $\gamma_{0}=1.0$, which are obtained based on performance on validation set after grid search hyperparameter optimization. The hyperparameter tuning is carried out on $\mathrm{KP} 20 \mathrm{~K}$ dataset and the best values are adopted for other datasets too. The value of $K$ is set to 2, which corresponds to upto 2-step ahead prediction.

For test time decoding, unlike previous work (Ye and Wang, 2018; Chen et al., 2019a; Yuan et al., 2020), we do not apply exhaustive decoding with large beam sizes, followed by pruning and de-duplication of the output. This is because our model is trained to generate outputs without repetitions. As such, we do not require any adhoc post-processing strategies to improve diversity. Thus we adopt greedy decoding at test time as well, similar to (Chan et al., 2019). For quality evaluation, we use the evaluation scripts ${ }^{7}$ provided by (Chan et al., 2019). Note that Porter Stemming is applied on the outputs for the purpose of quality evaluation.

\section{Results on Evaluation-Only Datasets}

We present additional results in Table 6 and Table 7 on the following datasets: INSPEC (Hulth and Megyesi, 2006), KRAP IVIN (Krapivin et al., 2009), NUS (Nguyen and Kan, 2007b), SEMEVAL (Kim et al., 2010) and DUC (Wan and Xiao, 2008). These datasets are smaller in size and hence, similar to previous work we only use them as test sets. DUC is a dataset with news articles and their associated

\footnotetext{
${ }^{6}$ Our initial experiments with Transformer-based architectures (Vaswani et al., 2017) showed similar performance, but required a lot more parameters. We thus carry out experiments with LSTM models as they are more parameter efficient and had lesser computational requirements. A similar observation has been reported in (Meng et al., 2019)

${ }^{7}$ https://github.com/kenchan0226/keyphrase-generation-rl
} 
keyphrases. Hence, we use the models trained on KPTimes for evaluation on DUC. Since the remaining datasets are from the domain of scientific articles, we test them using the best checkpoints obtained from training on KP $20 \mathrm{~K}$ dataset.

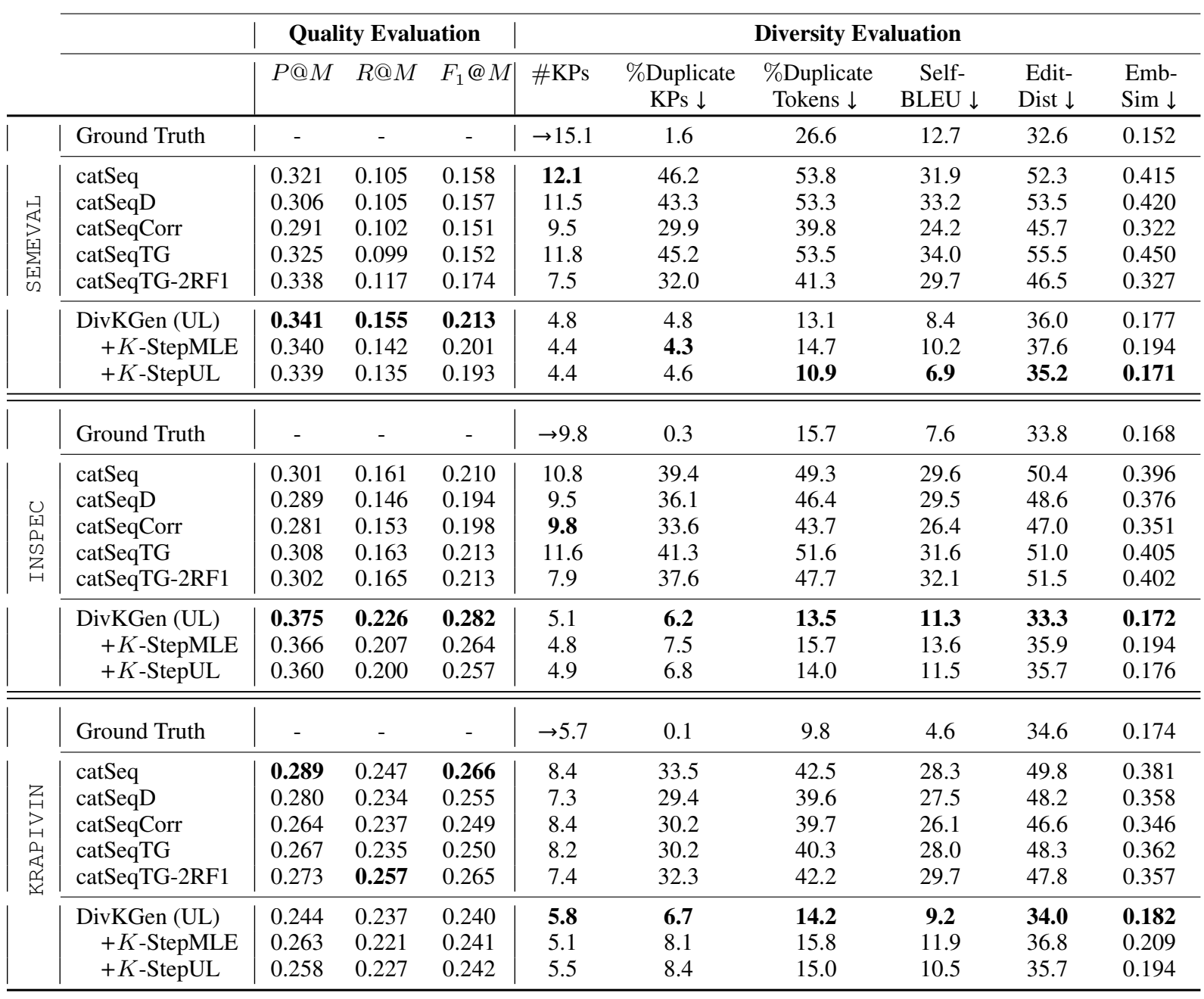

Table 6: Results of keyphrase generation on SEMEVAL, INSPEC and KRAP IVIN datasets. 


\begin{tabular}{|c|c|c|c|c|c|c|c|c|c|c|}
\hline & & \multicolumn{3}{|c|}{ Quality Evaluation } & \multicolumn{6}{|c|}{ Diversity Evaluation } \\
\hline & & $P @ M$ & $R @ M$ & $F_{1} @ M$ & \#KPs & $\begin{array}{c}\text { \%Duplicate } \\
\text { KPs } \downarrow\end{array}$ & $\begin{array}{c}\text { \%Duplicate } \\
\text { Tokens } \downarrow \\
\end{array}$ & $\begin{array}{c}\text { Self- } \\
\text { BLEU } \downarrow\end{array}$ & $\begin{array}{r}\text { Edit- } \\
\text { Dist } \downarrow \\
\end{array}$ & $\begin{array}{l}\text { Emb- } \\
\text { Sim } \downarrow \\
\end{array}$ \\
\hline \multirow{9}{*}{ 号 } & Ground Truth & - & - & - & $\rightarrow 11.7$ & 5.3 & 23.6 & 12.3 & 32.8 & 0.161 \\
\hline & catSeq & 0.391 & 0.210 & 0.274 & 11.7 & 43.6 & 52.0 & 31.6 & 53.7 & 0.442 \\
\hline & catSeqD & 0.397 & 0.206 & 0.271 & 10.4 & 41.4 & 49.6 & 32.2 & 52.9 & 0.433 \\
\hline & catSeqCorr & 0.396 & 0.217 & 0.281 & 10.7 & 38.9 & 47.8 & 29.8 & 50.1 & 0.398 \\
\hline & catSeqTG & 0.407 & 0.203 & 0.271 & 11.3 & 42.9 & 51.8 & 33.6 & 54.3 & 0.445 \\
\hline & catSeqTG-2RF1 & 0.385 & 0.228 & 0.286 & 7.6 & 32.6 & 44.1 & 30.0 & 47.4 & 0.355 \\
\hline & DivKGen (UL) & 0.376 & 0.238 & 0.292 & 5.3 & 6.5 & 15.0 & 10.3 & 34.8 & 0.189 \\
\hline & $+K$-StepMLE & 0.394 & 0.225 & 0.287 & 4.8 & 8.6 & 17.7 & 14.1 & 37.7 & 0.218 \\
\hline & $+K$-StepUL & 0.393 & 0.218 & 0.281 & 4.4 & 5.9 & 13.5 & 10.0 & 36.6 & 0.202 \\
\hline \multirow{9}{*}{$\begin{array}{l}\text { U } \\
?\end{array}$} & Ground Truth & - & - & - & $\rightarrow 8.1$ & 0.2 & 14.1 & 6.4 & 33.4 & 0.176 \\
\hline & catSeq & 0.106 & 0.059 & 0.076 & 5.9 & 19.5 & 28.5 & 24.6 & 38.0 & 0.243 \\
\hline & catSeqD & 0.104 & 0.057 & 0.074 & 6.2 & 20.5 & 29.8 & 24.8 & 38.1 & 0.249 \\
\hline & catSeqCorr & 0.103 & 0.057 & 0.073 & 5.5 & 15.0 & 24.9 & 20.3 & 36.8 & 0.226 \\
\hline & catSeqTG & 0.111 & 0.060 & 0.078 & 5.7 & 18.0 & 27.8 & 22.8 & 37.1 & 0.231 \\
\hline & catSeqTG-2RF1 & 0.115 & 0.069 & 0.086 & 6.2 & 19.4 & 28.9 & 27.1 & 36.2 & 0.217 \\
\hline & DivKGen (UL) & 0.135 & 0.065 & 0.088 & 4.2 & 3.4 & 9.5 & 5.6 & 30.4 & 0.151 \\
\hline & $+K$-StepMLE & 0.152 & 0.069 & 0.095 & 4.0 & 3.0 & 9.7 & 5.7 & 30.8 & 0.148 \\
\hline & $+K$-StepUL & 0.143 & 0.070 & 0.094 & 4.5 & 3.8 & 9.9 & 6.7 & 29.4 & 0.139 \\
\hline
\end{tabular}

Table 7: Results of keyphrase generation on NUS and DUC datasets.

\section{Qualitative Results}

In Tables 8, 9 and 10, we present qualitative results from the three domains respectively, i.e., scientific articles, news and community QA forums. The input to each model is the title and the abstract, and the expected output is displayed as the ground truth. In these case study examples, it can be observed that both the MLE and RL baseline tend to generate numerous repetitions in their output sequence. Our DivKGen base variant (UL) achieves good diversity, although occasionally it does generate few repetitions. However, we are able to avoid duplicates with the DivKGen (Full) model, which additionally incorporates the $K$-step ahead losses. We attribute this to be due to the enhanced model planning capabilities that DivKGen (Full) exhibits, by learning what the future tokens should or shouldn't be. 


\begin{tabular}{|c|c|}
\hline \multicolumn{2}{|r|}{ Dataset : KP20K } \\
\hline Title & automatic image segmentation by dynamic region merging . \\
\hline Abstract & $\begin{array}{l}\text { this paper addresses the automatic image segmentation problem in a region merging } \\
\text { style. with an initially oversegmented image, in which many regions or superpixels } \\
\text { with homogeneous color are detected, an image segmentation is performed by iteratively } \\
\text { merging the regions according to a statistical test . there are two essential issues in a region } \\
\text { merging algorithm order of merging and the stopping criterion . in the proposed algorithm } \\
\text { these two issues are solved by a novel predicate, which is defined by the sequential } \\
\text { probability ratio test and the minimal cost criterion. starting from an oversegmented } \\
\text { image, neighboring regions are progressively merged if there is an evidence for merging } \\
\text { according to this predicate. we show that the merging order follows the principle of } \\
\text { dynamic programming. this formulates the image segmentation as an inference problem } \\
\text { where the final segmentation is established based on the observed image . we also } \\
\text { prove that the produced segmentation satisfies certain global properties . in addition, a } \\
\text { faster algorithm is developed to accelerate the region merging process, which maintains a } \\
\text { nearest neighbor graph in each iteration. experiments on real natural images are conducted } \\
\text { to demonstrate the performance of the proposed dynamic region merging algorithm. }\end{array}$ \\
\hline Ground Truth & $\begin{array}{l}\text { image segmentation; region merging ; dynamic programming ; wald sequential probabil- } \\
\text { ity ratio test }\end{array}$ \\
\hline catSeq MLE Baseline & $\begin{array}{l}\text { image segmentation; region merging ; region merging ; dynamic programming ; image } \\
\text { segmentation }\end{array}$ \\
\hline catSeqTG-2RF1 (RL) & $\begin{array}{l}\text { image segmentation ; region merging ; dynamic programming ; image segmentation ; } \\
\text { dynamic programming }\end{array}$ \\
\hline DivKGen (UL) & $\begin{array}{l}\text { image segmentation ; region merging ; region merging ; dynamic programming ; nearest } \\
\text { neighbor graph }\end{array}$ \\
\hline DivKGen (Full) & image segmentation ; dynamic programming ; region merging ; stopping criterion \\
\hline
\end{tabular}

Table 8: Case Study on KP20K dataset. 


\begin{tabular}{|c|c|}
\hline \multicolumn{2}{|r|}{ Dataset : KPTimes } \\
\hline Title & n.f.l s said to be closer to testing for h.g.h . \\
\hline Abstract & $\begin{array}{l}\text { the n.f.l . owners and players have figured out how to divide up their money, and have } \\
\text { spent a busy week reconstituting rosters and renewing rivalries . but there is still unfinished } \\
\text { business in their labor standoff, and the most important issue remaining could be the } \\
\text { question of drug testing . the n.f.l . , whose new collective bargaining agreement is } \\
\text { expected to be completed and ratified by thursday, could begin blood testing for human } \\
\text { growth hormone as soon as september, according to a person briefed on the negotiations } \\
\text { who was not authorized to speak publicly, making it the first major north american sports } \\
\text { league to conduct such testing on its top players with the union consent . players had long } \\
\text { resisted blood testing under the former union president gene upshaw, and negotiators are } \\
\text { still determining ways to make the program acceptable to current players . details to be } \\
\text { worked out include how many players will be tested for performance enhancing drugs } \\
\text { and how they would be randomly selected when drug testing resumes . there was no drug } \\
\text { testing of any kind conducted during the lockout . but commissioner roger goodell and } \\
\text { demaurice smith, the players union executive director, were said by people briefed on } \\
\text { negotiations to have long seen the need for growth hormone testing and to want to cast } \\
\text { the n.f.l . as a leader in combating drugs in major sports . they have pointed to the joint } \\
\text { actions of upshaw and the former commissioner paul tagliabue, who moved to start the } \\
\text { steroid testing program in the late . i think both sides have a commitment to being leaders } \\
\text { in this area and to having the best }\end{array}$ \\
\hline Ground Truth & $\begin{array}{l}\text { human growth hormone ; goodell roger; national football league ; doping sports ; football } \\
\text {; organized labor ; smith demaurice ; tests drug use }\end{array}$ \\
\hline catSeq MLE Baseline & $\begin{array}{l}\text { human growth hormone; national football league; football ; tests and testing ; national } \\
\text { football league ; tests drug use; tests drug use; national football league ; tests drug use ; } \\
\text { doping sports ; tests drug use; national football league; tests drug use }\end{array}$ \\
\hline catSeqTG-2RF1 (RL) & $\begin{array}{l}\text { human growth hormone; baseball ; national football league; tests drug use ; national } \\
\text { football league ; football ; national football league ; lockouts; organized labor }\end{array}$ \\
\hline DivKGen (UL) & $\begin{array}{l}\text { human growth hormone; drug abuse and traffic; national football league; goodell roger } \\
\text {; lockouts; national football league }\end{array}$ \\
\hline DivKGen (Full) & $\begin{array}{l}\text { human growth hormone ; upshaw gene ; goodell roger ; national football league ; } \\
\text { organized labor; lockouts ; football }\end{array}$ \\
\hline
\end{tabular}

Table 9: Case Study on KPTimes dataset.

\begin{tabular}{|l|l|}
\hline Title & \multicolumn{1}{c|}{ Dataset : StackEx } \\
\hline Abstract & $\begin{array}{l}\text { do deep learning algorithms represent ensemble based methods ? } \\
\text { based on a set of algorithms that attempt to model high level abstractions in data by } \\
\text { using a deep graph with multiple processing layers, composed of multiple linear and non } \\
\text { linear transformations.various deep learning architectures such as deep neural networks , } \\
\text { convolutional deep neural networks, deep belief networks and recurrent neural networks } \\
\text { have been applied to fields like computer vision, automatic speech recognition, natural } \\
\text { language processing, audio recognition and bioinformatics where they have been shown } \\
\text { to produce state of the art results on various tasks.my question can deep neural networks } \\
\text { or convolutional deep neural networks be viewed as ensemble based method of machine } \\
\text { learning or it is different approaches }\end{array}$ \\
\hline Ground Truth & deep learning; machine learning ; neural networks; convolutional neural networks \\
\hline \hline catSeq MLE Baseline & deep learning; machine learning; deep learning \\
\hline catSeqTG-2RF1 (RL) & deep learning; machine learning; neural network; machine learning \\
\hline DivKGen (UL) & deep learning ; machine learning; ensemble modeling \\
\hline DivKGen (Full) & deep learning; neural networks \\
\hline
\end{tabular}

Table 10: Case Study on StackEx dataset. 